\title{
Lymphopenia: A useful predictor of COVID-19 disease severity and mortality
}

\author{
Kaleem Ullah Toori ${ }^{1}$, \\ Muhammad Arsalan Qureshi², Asma Chaudhry ${ }^{3}$
}

\begin{abstract}
Objective: To detect association of lymphopenia with disease severity and mortality.

Methods: Total 874 COVID RT-PCR positive patients admitted to KRL Hospital Islamabad from April 2020 to August 2020 were included in this cross-sectional study. Lymphopenia was defined as $<1100$ cells/micro-L. WHO categories for disease severity were used. Demographic profile, absolute lymphocyte counts and comorbidities were recorded. Pearson's Chi Square test was used to see association between lymphopenia and disease severity as well as disease outcome. Regression analysis was used to see whether lymphopenia would predict disease severity. Comparison of means of absolute lymphocyte count in different disease categories was done by ANOVA. Tukey's test range was then used to find the means different from each other. P-value $\leq 0.05$ was considered statistically significant

Results: The mean age of patients was $40 \pm 12.3$ years. Majority patients (73.9\%) were asymptomatic. Lymphopenia was present in $6.9 \%$ of total patients. Significant association was found between lymphopenia and disease severity as well as lymphopenia and mortality $(<0.001)$. Lymphopenia was found to be a predictor of disease severity using regression analysis $(<0.001)$. Comparison of mean absolute lymphocyte count was significant among disease severity categories $(<0.001)$. On post-hoc analysis, difference in absolute lymphocyte count was significant moving from asymptomatic to mild and then moderate disease category. However no significant difference was seen in absolute lymphocyte count between moderate and severe categories.

Conclusion: Results are compatible with worldwide studies and lymphopenia is valid as a marker of disease severity and mortality.
\end{abstract}

KEYWORDS: COVID-19 RT-PCR, Disease severity (asymptomatic, mild, moderate and severe), Lymphopenia.

How to cite this:

doi: https://doi.org/10.12669/pjms.37.7.4085

Toori KU, Qureshi MA, Chaudhry A. Lymphopenia: A useful predictor of COVID-19 disease severity and mortality. Pak J Med Sci. 2021;37(7):1984-1988. doi: https://doi.org/10.12669/pjms.37.7.4085

This is an Open Access article distributed under the terms of the Creative Commons Attribution License (http://creativecommons.org/licenses/by/3.0), which permits unrestricted use, distribution, and reproduction in any medium, provided the original work is properly cited.

1. Dr. Kaleem Ullah Toori, FRCP (Glasgow).

2. Dr. Muhammad Arsalan Qureshi (M.B.B.S).

3. Dr. Asma Chaudhry, MRCP (UK), FCPS General Medicine (Pakistan). Department of Medicine and Endocrinology,

Southend University Hospital,

Southend-on-Sea, United Kingdom.

1,2: Department of Medicine,

KRL Hospital, Islamabad, Pakistan.

Correspondence:

Dr. Kaleem Ullah Toori,

Consultant Physician and Pulmonologist,

KRL Hospital,

Islamabad, Pakistan.

Email: kaleem_toori@msn.com

* Received for Publication:

* Revision Received:

* Revision Accepted:

December 26, 2020

June 16, 2021

July 8, 2021

\section{INTRODUCTION}

Corona virus disease (COVID-19) first emerged from Wuhan in China and spread rapidly to different parts of the world. It was declared a pandemic by the World Health Organization (WHO) on March $11,2020 .{ }^{1}$ The virus has affected millions of people.

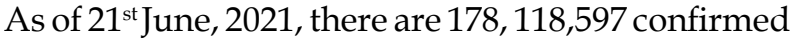
cases of the virus and more than 3,864,180 deaths that have been reported to the WHO. ${ }^{2}$ Pakistan's index cases was reported back in February 2020 in Karachi and Rawalpindi. ${ }^{3}$ The number of cases in the country rose to 948,268 with almost 22,000 deaths in June, 2021. ${ }^{4}$ As Mass vaccination drive 
have started throughout the country after approval of multiple Covid-19 vaccines, it can be expected that the course of the disease may change.

It has been postulated that the spread of disease was mainly by human-to-human contact. ${ }^{5}$ The signs and symptoms of the disease are not limited to a particular body system, rather a range of presentations occur, requiring isolation in asymptomatic or mild cases and oxygen, mechanical ventilation and ICU care with more severe presentation. ${ }^{6}$ The $\mathrm{WHO}$ has categorized the disease as asymptomatic, mild, moderate, severe and critical. Case positivity is defined by positive RT-PCR regardless of presence or absence of symptoms. ${ }^{7}$

Researchers have tried to come up with possible immunological parameters that might help to predict the severity of disease or a clinical outcome. Many hematological parameters are currently under interest to predict outcomes and mortality. Lymphocyte count has been of keen interest to clinicians all over the world probably as it is economical and easily available. Numerous studies all over the world ${ }^{8-12}$ have revealed that lymphopenia is an immunological finding in patients infected with corona virus and may be used as a prognostic marker. It is also a marker that predicts mortality amongst critically ill COVID-19 patients.

The aim of this study was to see whether a simple investigation like reduced lymphocyte count was associated with COVID-19 disease severity and mortality. We wanted to identify if lymphopenia could be used as a predictor of disease severity. We also wanted to see how the absolute lymphocyte count would vary amongst disease severity categories. Methods to achieve this are mentioned in detail below. Limited data is available regarding these parameters in the region as COVID-19 is still an emerging pandemic.

\section{METHODS}

This was a hospital-based, descriptive crosssectional study, conducted at KRL hospital Islamabad where a consecutive series of patients $1^{\text {st }}$ April 2020 to $31^{\text {st }}$ August 2020 were studied. Sample size calculation was not applicable as there is no data on prevalence of the disease in Pakistani population or internationally. ${ }^{13}$ The study was started after taking approval from the hospital's ethical and research committee (Ref ERC: KRL-HI-ERC/Dec20/27, dated March 30, 2020). All patients were employees of the
KRL organization. Our organization developed an isolation facility for the COVID-19 positive patients. Written informed consent was taken from all patients.

Patients who presented with possible signs and symptoms of COVID-19 were first isolated and Reverse transcription polymerase chain reaction (RT-PCR) of nasopharyngeal swabs was performed. Those with a positive RT-PCR were admitted and included in the study. History, demographic profile, exposure, drug history, comorbidities and relevant medical details were obtained and recorded. Investigations including blood tests and radiology were performed as per hospital's protocol. All X-rays and CT scans were reported by a classified radiologist. Lymphopenia was calculated using Complete Blood Count with differential count. The definition of Lymphopenia adapted is $<1100$ cells/micro-L. ${ }^{8}$ Lymphopenia was documented on day of admission and then serial testing during stay at the hospital.

Case severity definitions were as per the interim guidance of WHO. ${ }^{7}$ Asymptomatic (patients are RT-PCR positive but do not show symptoms), mild (patients are RT-PCR positive but no hypoxia), moderate (RT-PCR positive patients who show signs of pneumonia and but no signs of severe hypoxia with Spo2 > 90\%), severe (signs of severe pneumonia evident with respiratory rate more than 30 breaths/minute or Spo $2<90 \%$ and critical (with Acute Respiratory Distress Syndrome [ARDS] or septic shock). We included both severe and critical patients under category of severe.

Statistical Analysis: Statistical analysis was performed using IBMSPSS 25. Continuous variables like age were expressed as mean values with standard deviation. Frequency and percentages were calculated for categorical variables like cases as per severity, symptoms, comorbidities and lymphopenia. Categorical variables were tested using Pearson's Chi square test to check for statistical significance. Comparison of mean values was done by one way Anova test and Tukey's range test was used to find the means different from each other. Simple regression analysis was applied to determine whether lymphopenia would be a predictor of disease severity. P-value $\leq 0.05$ was considered statistically significant. Confidence interval was at $95 \%$.

\section{RESULTS}

Total 874 patients were analysed during this study. The mean age of patients was $40 \pm 12$.3 years 
Table-I: Association between disease severity and lymphopenia- Pearson Chi square and Regression Analysis.

\begin{tabular}{lccc}
\hline Disease Severity Categories & Lymphopenia $(\%)$ & Pearson's $\chi^{2}$ value $(p)[$ d.o.f] & Regression \\
\hline Asymptomatic & $19(2.9 \%)$ & & \\
Mild & $7(4.8 \%)$ & $183.36(<0.001)[3]$ & $\mathrm{R}^{2}=0.17, \mathrm{~F}(1,869)=$ \\
Moderate & $12(30.8 \%)$ & & \\
Severe & $22(51.2 \%)$ & & \\
\hline
\end{tabular}

d.o.f: Degree of freedom.

(range 12 - 87 years). Majority were males i.e., 96\% with only $4 \%$ being female. Out of the total patients admitted, $73.9 \% \quad(\mathrm{n}=646)$ were asymptomatic while $26.1 \%(\mathrm{n}=228)$ were symptomatic. Among the symptomatic, the most frequent symptoms were fever $87.3 \%(n=199)$, cough $54.8 \%(n=125)$, dyspnoea $32 \%(n=73)$, myalgia $28.5 \%(n=65)$, sore threat $16.7 \%(\mathrm{n}=38)$, diarrhoea and vomiting $5.2 \%$ $(n=12)$ headache $3.1 \%(n=7)$, rhinorrhoea $2.2 \%$ $(n=5)$ and haemoptysis $0.41 \%(n=1)$, in order of decreasing frequency.

On reviewing co-morbidities, $84 \%(\mathrm{n}=734)$ of patients had no pre-morbidity while $16 \%(\mathrm{n}=140)$ had underlying chronic medical conditions. The reported pre-morbid conditions were diabetes mellitus in $8.7 \% \quad(\mathrm{n}=76)$, hypertension in $8.2 \%$ $(n=72)$, ischemic heart disease in $2.9 \% \quad(n=25)$, chronic respiratory disease in $2.5 \%(\mathrm{n}=22)$, chronic kidney disease in $1.7 \%(\mathrm{n}=15)$, chronic liver disease in $0.5 \%(n=4)$, chronic neuro-psychiatric condition in $0.2 \%(n=2)$ and malignancy $0.1 \%(n=1)$

Regarding disease severity, $73.9 \%(n=646)$ were asymptomatic, $16.7 \%(\mathrm{n}=146)$ had mild disease, $4.5 \%(n=39)$ had moderate disease and $4.9 \%(n=43)$ had severe disease. $98.2 \% \quad(\mathrm{~N}=858)$ of patients recovered while $1.8 \%(n=16)$ of patients died.
Lymphopenia was present in $6.9 \% \quad(n=60)$ of total patients. Association between disease severity and lymphopenia is reported in Table-I. Lymphopenia was more frequent in moderate and severe disease, which was significant by applying Pearson Chi square proving a positive association of lymphopenia with disease severity. Using regression analysis we also found that lymphopenia was a significant predictor of disease severity, the results are reported in Table-I.

Further analysis was done to compare the means of absolute lymphocyte count amongst disease severity categories using ANOVA. The comparison of means was significant as reported in Table-II. We then applied the Tukey's range test to find the means that were significantly different from each other. The difference in mean lymphocyte count is statistically significant from asymptomatic to mild, and mild to moderate categories. However no significant difference was noted in moderate to severe category as reported in Table-III.

Among the patients $(\mathrm{n}=16)$ who died of COVID-19, majority (56.3\%) were found to have lymphopenia. The recovered group had fewer cases of lymphopenia. Pearson Chi square test was used to test the association between mortality and

Table-II: Variability of absolute lymphocyte count across all categories of disease severity- ANOVA.

\begin{tabular}{lccccc}
\hline & & \multicolumn{4}{c}{ Disease Severity } \\
\cline { 3 - 6 } & & Asymptomatic & Mild & Moderate & Severe \\
\hline & Mean & 2542.5 & 2143.5 & 1471.3 & 1121.2 \\
& $(95 \% \mathrm{CI})$ & $(2465,2620)$ & $(2004,2282)$ & $(1190,1752)$ & $(989,1253)$ \\
\hline Absolute & Minimum & 572 & 744 & 408 & 510 \\
Cymphocyte & Maximum & 6888 & 4074 & 4448 & 2340 \\
& $\mathrm{SD}$ & 39.4 & 70 & 138 & 65 \\
& $\mathrm{~N}$ & 551 & 96 & 33 & 40 \\
\hline
\end{tabular}

CI: Confidence interval, SD: Standard Deviation, N: Number of individuals. 
Kaleem Ullah Toori et al.

Table-III: Difference in mean absolute lymphocyte range amongst disease severity- Tukey's range test

\begin{tabular}{lccccc}
\hline & Mean Difference & Standard Error & p-value & Lower bound & Upper bound \\
\hline Asymptomatic- Mild & $398.97^{*}$ & 96.28 & $<0.001$ & 151.03 & 646.92 \\
Mild-Moderate & $672.22^{*}$ & 175.68 & 0.001 & 219.83 & 1124.62 \\
Moderate-Severe & 350.08 & 204.73 & 0.319 & -177.13 & 877.30 \\
\hline
\end{tabular}

Table-IV: Frequency between lymphopenia and mortality- Pearson Chi square.

\begin{tabular}{lcc}
\hline Outcome & $\begin{array}{c}\text { Lymphopenia } \\
n(\%)\end{array}$ & $\begin{array}{c}\text { Pearson's } \chi^{2} \text { value }(p) \\
{[\text { d.o.f] }}\end{array}$ \\
\hline Recovered & $51(5.9 \%)$ & $62.17(<0.001)[1]$ \\
Died & $9(56.3 \%)$ & \\
\hline
\end{tabular}

d.o.f: degree of freedom.

lymphopenia, reported in Table-IV. This reveals a significant association between mortality and lymphopenia.

\section{DISCUSSION}

COVID-19 is an emerging pandemic which has caused havoc around the world. A lot of investigations are ongoing to identify disease markers of severity and mortality. Lymphocyte count is one of the haematological markers under investigation. Lymphopenia occurs due to a variety of reasons in COVID-19 patients. Cytokine storm is a phenomenon of excessive inflammatory reaction and has been associated with lymphopenia, with greater levels of inflammatory markers (TNF alpha and Interleukin 6 [IL-6]) causing more lymphocyte death. Mortality in COVID-19 patients was also linked with lower lymphocyte count in American studies. ${ }^{10}$ According to Mazzoni et al. ${ }^{11}$, high IL-6 levels lead to impaired immune cytotoxic activity of lymphocytes which are necessary to fight against viral illnesses. COVID-19 may also result in T cell exhaustion via increased expression of certain cell surface proteins with one Chinese study concluding that markers of $\mathrm{T}$ cell exhaustion are over expressed in COVID-19 patients in the ICU. ${ }^{12}$

The mean age of patients developing COVID-19 is variable. The mean age in our population was 40 years which is lower as compared to other Pakistani studies. ${ }^{14,15}$ Our patients were predominantly males which is keeping with other studies as COVID-19 is known to have a predilection for male gender. ${ }^{14,15}$

Majority patients in our study were asymptomatic. This is similar to data acquired by
WHO which suggests up to eighty percent patients maybe asymptomatic. ${ }^{16}$ Amongst the symptomatic individuals, the most common clinical symptoms were fever, respiratory symptoms, and myalgias which are similar to those described by the $\mathrm{WHO}$ interim guidance. ${ }^{7}$

The study at our center has shown significant results with regards to relationship of lymphopenia and disease severity. Moderate and severe cases had statistically significant lymphopenia. We also found that as the disease severity increases from asymptomatic to mild and then moderate disease, the absolute lymphocyte count falls steadily and significantly. As disease progressed from moderate to severe, there was a fall in absolute lymphocyte counts, however this was not significant. We attributed this non-significance to very small number of patients in both moderate $(4.5 \%)$ and severe $(4.9 \%)$ categories. This difference might be better evident if greater numbers of moderate and severe patients would be included in subsequent studies. Lymphopenia in COVID-19 has been a well-documented finding in international studies. ${ }^{10-12}$ The results of our study are seconded by a Korean study which concluded that lymphopenia was more prominent in severe cases as compared to mild cases. ${ }^{17}$ Pakistani studies also documented significant lymphopenia in Intensive Care Unit patients. ${ }^{14,15}$

We also established a link between lymphopenia and mortality associated with COVID-19 patients. Meta-analysis of 23 studies involving more than 3000 patients also confirmed that greater degree of lymphopenia is associated with poor clinical outcome and that those who died had lower lymphocyte counts which was statistically significant; further endorsing what our study has shown. ${ }^{8}$

The results of our study are consistent with the above-mentioned studies which supports the hypothesis that lymphopenia is an important indicator and degree of lymphopenia can be used as a marker of disease severity. Our study further adds to the evidence that lymphopenia 
correlates with severe and deteriorating COVID-19 infection in the Pakistani population. It can provide an objective sign to identify patients at risk of deterioration early, leading to improved management of critical patients.

Limitations of the study: The major limitations in our study were the predominant male number patients as they were employees of the organization and were required to be admitted if they were positive for RT-PCR -2. On the other hand, female patients opted to isolate at home unless they had moderate to severe disease requiring hospital management. Secondly, this was a single-center study.

\section{CONCLULSION}

The lymphocyte count is a simple, effective and cheap guide to predict disease severity and mortality outcome amongst COVID-19 patients. This is especially important in our country, where lack of resources may limit expensive investigations. Earlier detection of lymphopenia should alert physicians to monitor for deterioration and investigate appropriately.

\section{Grant Support E Financial Disclosures: None.}

\section{REFERENCES}

1. Cucinotta D, Vanelli M. WHO declares COVID-19 a pandemic. Acta Biomed. 2020;91(1):157-160. doi: 10.23750/ abm.v91i1.9397

2. World Health Organization. WHO Coronavirus Disease (COVID-19) Dashboard[Internet] Covid19.Who.int.com. 2021. [Cited 21 June 2021] Available from: https:/ /covid19. who.int/ ?adgroupsurvey $=\{$ adgroupsurvey $\}$ \&gcli$\mathrm{d}=$ CjwKCAjw8cCGBhB6EiwAgOReyzuxoYbnFfeYzoj0 ADxbRZLSiYaKseOmNR9RuDTbzGc7oT_KI58vRoCrb4QAvD_BwE

3. World Health Organization. WHO extends support to Pakistan as it confirms its first two cases of Covid-19 [Internet] emro.who.int.com. 2020. [Cited 12 November 2020] Available from: http://www.emro.who.int/pak/ pakistan-news / who-extends-support-to-pakistan-as-itconfirms-its-first-two-cases-of-covid-19.html

4. Government of Pakistan. Pakistan Statistics [Internet] covid. gov.pk. 2021 [Cited 21 June 2021] Available from: http:// covid.gov.pk/

5. World Health Organization. Origin of SARS-CoV2[Internet] who.int.com. 2020. [Cited 12 November 2020] Available from: https://apps.who.int/iris/bitstream/ handle/10665/332197/WHO-2019-nCoV-FAQ-Virus_origin-2020.1-eng.pdf

6. Huang C, Wang Y, Li X, Ren L, Zhao J, Hu Y, et al. Clinical features of patients infected with 2019 novel coronavirus in Wuhan, China. Lancet. 2020;395(10223):497-506. doi: 10.1016/S0140-6736(20)30183-5
7. World Health Organization. Clinical management of COVID-19: interim guidance May 2020 [Internet] Who.int.com. 2020. [Cited 12 November 2020] Available from: https:// www.who.int/publications/i/item/clinical-managementof-covid-19

8. Huang I, Pranata R. Lymphopenia in severe coronavirus disease-2019 (COVID-19): Systematic review and metaanalysis. J Intensive Care. 2020;8(36):1-10. doi: 10.1186/ s40560-020-00453-4

9. Wang Z, Yang B, Li Q, Wen L, Zhang R. Clinical features of 69 cases with coronavirus disease 2019 in Wuhan, China. Clin Infect Dis. 2020;71(15):769-777. doi: 10.1093/ cid/ciaa272

10. Tavakolpour S, Rakhshandehroo T, Wei EX, Rashidian M. Lymphopenia during the COVID-19 infection: What it shows and what can be learned. Immunol Lett. 2020;225:3132. doi: 10.1016/j.imlet.2020.06.013

11. Mazzoni A, Salvati L, Maggi L, Capone M, Vanni A, Spinicci $\mathrm{M}$, et al. Impaired immune cell cytotoxicity in severe COVID-19 is IL-6 dependent. J Clin Invest. 2020;130(9):4694-4703.

12. Diao B, Wang C, Tan Y, Chen X, Liu Y, Ning L, et al. Reduction and functional exhaustion of $\mathrm{T}$ cells in patients with coronavirus disease 2019 (COVID-19). Front Immunol. 2020;11(827):1-7. doi: 10.3389/ fimmu.2020.00827

13. Guan WJ, Liang WH, Zhao Y, Liang HR, Chen ZS, Li YM, et al. Comorbidity and its impact on 1590 patients with COVID-19 in China: A nationwide analysis. Eur Respir J. 2020;55(2000547):1-14. doi: 10.1183/13993003.00547-2020

14. Asghar MS, Kazmi SJH, Khan NA, Akram M, Khan SA, Rasheed U, et al. Clinical Profiles, Characteristics, and Outcomes of the First 100 Admitted COVID-19 Patients in Pakistan: A Single-Center Retrospective Study in a Tertiary Care Hospital of Karachi. Cureus. 2020;12(6):e8712. doi: 10.7759/ cureus.8712

15. Asghar MS, Khan NA, Kazmi SJH, Ahmed A, Hassan M, Jawed R, et al. Hematological parameters predicting severity and mortality in COVID-19 patients of Pakistan: a retrospective comparative analysis. J Hosp Intern Med Perspect. 2020;10(6):514-520. doi: 10.1080/20009666.2020.1816276

16. World Health Organization. Coronavirus disease (COVID-19): Similarities and differences with influenza [Internet] Who.int.com. 2020. [Cited 12 November 2020] Available from: https:// www.who.int/emergencies/diseases/novel-coronavirus-2019/question-and-answershub/q-a-detail/q-a-similarities-and-differences-covid19-and-influenza

17. Kim ES, Chin BS, Kang CK. Clinical course and outcomes of patients with severe acute respiratory syndrome coronavirus 2 infection: A preliminary report of the first 28 patients from the Korean cohort study on COVID-19. J Korean Med Sci. 2020;35(13):e142.

\section{Authors`Contribution:}

KT conceived, designed and did statistical analysis along with review of manuscript and accountable for the accuracy of the study.

MAQ contributed to data collection and manuscript writing.

AC did manuscript writing with final review of manuscript. 